\title{
Thermodynamical and dynamical stability of a self-gravitating uncharged thin shell
}

\author{
Santiago Esteban Perez Bergliaffa ${ }^{1, a}$, Marcelo Chiapparini ${ }^{1, b}$, Luz Marina Reyes $^{2, \mathrm{c}}$ \\ ${ }^{1}$ Departamento de Física Teórica, Instituto de Física, Universidade do Estado do Rio de Janeiro, Rua São Francisco Xavier 524, Maracanã, \\ Rio de Janeiro CEP 20550-900, Brasil \\ ${ }^{2}$ Departamento de Ciencias Computacionales, CUCEI, Universidad de Guadalajara, Av. Revolución 1500, 44430 Guadalajara, Jal, México
}

Received: 13 April 2020 / Accepted: 21 July 2020 / Published online: 10 August 2020

(C) The Author(s) 2020

\begin{abstract}
The dynamical stability of massive thin shells with a given equation of state (EOS) (both for the barotropic and non-barotropic case) is here compared with the results coming from thermodynamical stability. Our results show that the restrictions in the para-meter space of equilibrium configurations of the shell following from thermodynamical stability are much more stringent that those obtained from dynamical stability. As a byproduct, we furnish evidence that the link between the maximum mass along a sequence of equilibrium configurations and the onset of dynamical stability is valid for EOS relating the pressure $P$, the energy density $\sigma$ of the matter on the shell, and its radius $R$, namely $P=P(R, \sigma)$.
\end{abstract}

\section{Introduction}

Self-gravitating thin shells are solutions of a given gravitational theory describing two regions separated by an infinitesimally thin region where matter is confined. Such a system conjugates the notions of vacuum, typical of black holes, together with the presence of matter, which may be described via statistical mechanics and thermodynamics. Thin shells have been frequently employed to probe thermodynamical properties of black holes, see for instance [14-17,24] and, by taking them to their own gravitational radius, they can be transformed into quasi-black holes [18], and used to calculate black hole properties (see for instance [12]). ${ }^{1}$ In view of these applications, it is important to determine whether relevant thin-shell configurations are stable, both thermo-

\footnotetext{
${ }_{1}^{1}$ For a complete list of applications of the thin-sell formalism see [9].

a e-mail: sepbergliaffa@gmail.com

be-mail: chiapparini.uerj@gmail.com

c e-mail: luzmarinareyes@gmail.com (corresponding author)
}

dynamically and dynamically. The thermodynamic stability of a spherically symmetric thin shell in which the interior region is Minkowski's spacetime and the exterior given by Schwarzschild's geometry was studied in [20], while the linear dynamical stability of such systems under radial perturbations was analyzed in [2] for a linear EOS, and in [7] for a general EOS. Since these two types of stability yield inequivalent restrictions on the parameter space [20], ${ }^{2}$ we shall present here the results of imposing both types of stability on a neutral thin shell configuration, for different barotropic equations of state, and also for EOS of the type $P=P(R, \sigma)$. The latter have been used in various settings such as wormholes (see for instance [5,21,23]), stars in Eddington-inspired BornInfeld gravity [10] and gravastars [19], and in cosmology $[4,6]$, among others. For a given EOS, we shall determine the region of the parameter space of equilibrium configurations of the shell compatible with both types of stability, and with the dominant energy condition (DEC). As a byproduct, we shall obtain evidence supporting the extension of the results linking the maximum mass with the onset of instability [11] to EOS of the type $P=P(R, \sigma)$.

The paper is organized as follows. In Sect. 2 we shall present a brief review of the relevant equations for the thin shell in equilibrium (mostly following [15]). The equations obeyed by the perturbed shell for an EOS of the type $P=P(R, \sigma)$ will be introduced in Sect. 3. In Sect. 4 we shall present the analysis of the dynamical stability of the shells for different barotropic EOS, along with the corresponding mass-radius relation $m=m(R)$. Section 5 is devoted to a non-barotropic EOS. The restrictions following from thermodynamical stability are exhibited in Sect. 6. In Sect. 7 we determine the set of equilibrium states of the shell that are

\footnotetext{
$\overline{2}$ See [22] for the relation between microcanonical thermodynamical stability and linear dynamical stability in the case of a static, perfect fluid sphere with a general equation of state in General Relativity.
} 
both dynamically and thermodynamically stable. Our closing remarks are presented in Sect. 8.

\section{Junction conditions and properties of the thin-shell}

Let us consider a two-dimensional timelike massive shell $\Sigma$ with radius $R$. The shell divides the spacetime in two parts: i) an inner region $r<R$, with flat geometry, and ii) an outer region $r>R$, in which the geometry is described by the Schwarzschild line element. In this way, we can express the metric in both regions as follows:

$d s_{I}^{2}=-f_{I} d t_{I}^{2}+g_{I} d r^{2}+r^{2} d \Omega^{2}$.

Here $I=o, i$ refers either to the outer or inner region, and the functions $f_{I}$ and $g_{I}$ are given by

$f_{i}=g_{i}=1, \quad f_{o}=1-\frac{2 m}{r}, \quad g_{o}=\left(1-\frac{2 m}{r}\right)^{-1}$,

where $m$ is the ADM mass, $d \Omega^{2} \equiv d \theta^{2}+\sin \theta^{2} d \phi^{2}$.

The metric $h_{a b}$, defined on $\Sigma$, i.e. for $r=R$, is that of a 2 -sphere, and can be written as

$d s_{\Sigma}^{2}=h_{a b} d y^{a} d y^{b}=-d \tau^{2}+R^{2}(\tau) d \Omega^{2}$,

where $y^{a}=(\tau, \theta, \phi)$ and $R$ is a function of $\tau$, the proper time for an observer located on the shell, in the dynamical case. The application of the thin-shell formalism developed in [8] to join the two spacetimes specified in Eqs. (1) and (2) requires the induced metric $h_{a b}$ to be continuous on the shell, and the discontinuity in the extrinsic curvature to be proportional to the stress-energy tensor on the shell, denoted by $\mathcal{S}_{\nu}^{\mu}$. The latter is given by the surface energy density $\sigma$ and the tangential pressure $p$ which, for a static shell, are as follows (see for instance [20]):

$$
\begin{gathered}
\mathcal{S}^{\tau}{ }_{\tau} \equiv \sigma_{0}=\frac{1-\sqrt{1-\frac{2 m_{0}}{R_{0}}}}{4 \pi R_{0}}, \\
\mathcal{S}^{\theta}{ }_{\theta}=\mathcal{S}^{\phi}{ }_{\phi} \equiv P_{0}=\frac{\sqrt{1-\frac{2 m_{0}}{R_{0}}-1}}{8 \pi R_{0}}+\frac{m_{0}}{8 \pi R_{0}^{2} \sqrt{1-\frac{2 m_{0}}{R_{0}}}},
\end{gathered}
$$

where the subindex 0 means that the quantities are evaluated at the equilibrium configuration.

The proper mass of the shell, denoted by $M$, is given by $M=4 \pi R_{0}^{2} \sigma_{0}$. The junction conditions also imply that the ADM mass is given by

$m_{0}=M-\frac{M^{2}}{2 R_{0}}$
Hence we can write

$$
\begin{aligned}
& P_{0}\left(M, R_{0}\right)=\frac{M^{2}}{16 \pi R_{0}^{2}\left(R_{0}-M\right)}, \\
& \sigma_{0}\left(M, R_{0}\right)=\frac{M}{4 \pi R_{0}^{2}} .
\end{aligned}
$$

We shall assume that $\sigma_{0}$ and $P_{0}$ are non-negative (hence $M>$ $0)$ and $R_{0} \geq 2 m_{0} \cdot^{3}$

Notice that by inverting Eqs. (7) and (8) we obtain $R_{0}\left(P_{0}, \sigma_{0}\right)$ and $M\left(P_{0}, \sigma_{0}\right)$ respectively given by

$$
\begin{aligned}
& R_{0}\left(P_{0}, \sigma_{0}\right)=\frac{P_{0}}{\pi \sigma_{0}\left(\sigma_{0}+4 P_{0}\right)}, \\
& M\left(P_{0}, \sigma_{0}\right)=\frac{4 P_{0}^{2}}{\pi \sigma_{0}\left(\sigma_{0}+4 P_{0}\right)^{2}} .
\end{aligned}
$$

Using Eqs. (6) and (10) it follows that

$m_{0}\left(M, R_{0}\right)=4 \pi \sigma_{0} R_{0}^{2}\left(1-2 \pi \sigma_{0} R_{0}\right)$.

Equations (9) and (11) describe the mechanical equilibrium of the shell, and will be used in the analysis of the linear stability and to build the $m_{0}=m_{0}\left(R_{0}\right)$ diagrams for a given EOS, as we shall see in Sect. 4.

Before moving to the dynamical stability of the shell, let us introduce the redshift of the shell, defined as

$k=\sqrt{1-\frac{2 m_{0}}{R_{0}}}$.

It follows that $M=R_{0}(1-k)$.

\section{Dynamical stability}

We shall outline here the steps that lead to the condition for the linear dynamical stability of the shells introduced in the previous section. While Eqs. (7) and (8) describe the equilibrium state of the shell, the corresponding expressions for a dynamical shell are (see for instance $[5,7])^{4}$

$$
\begin{aligned}
\sigma= & \frac{1}{4 \pi} \frac{\sqrt{f_{o}(R)+\dot{R}^{2}}-\sqrt{f_{i}(R)+\dot{R}^{2}}}{R}, \\
P= & \frac{1}{8 \pi}\left[\frac{2 \ddot{R}+f_{o}^{\prime}(R)}{2 \sqrt{f_{o}(R)+\dot{R}^{2}}}-\frac{2 \ddot{R}+f_{i}^{\prime}(R)}{2 \sqrt{f_{o}(R)+\dot{R}^{2}}}\right. \\
& \left.+\frac{\sqrt{f_{o}(R)+\dot{R}^{2}}-\sqrt{f_{i}(R)+\dot{R}^{2}}}{R}\right],
\end{aligned}
$$

\footnotetext{
${ }^{3}$ We use units such that $[M]=[m]=[R]=L$ and $[P]=[\sigma]=1 / L$.

${ }^{4}$ For a derivation of the dynamics of the shell following a Hamiltonian treatment, see [3].
} 
where $f_{i}(R)$ and $f_{o}(R)$ are defined in Eq. (2), and the overdot denotes the derivative with respect to $\tau$. These quantities obey the equation that follows from the conservation of $\mathcal{S}_{\nu}^{\mu}$, namely

$\frac{d \sigma}{d R}+\frac{2}{R}(P+\sigma)=0$.

A radial perturbation of an equilibrium configuration with $R=R_{0}$ causes $R, \sigma$ and $P$ to become functions of $\tau$. Assuming an EOS of the type $P=P(R, \sigma)$, it follows from Eqs. (13) and (15) that the evolution of the shell is governed by the equation [7]

$\dot{R}^{2}+V(R, \sigma(R))=0$,

where

$$
\begin{aligned}
V(R)= & \frac{1}{2}\left(f_{i}(R)+f_{o}(R)\right)-\frac{1}{64} \frac{\left(f_{i}(R)-f_{o}(R)\right)^{2}}{\pi^{2} R^{2} \sigma(R)^{2}} \\
& -4 \pi^{2} R^{2} \sigma(R)^{2} .
\end{aligned}
$$

The linear stability of the shell can be studied by expanding the potential $V(R)$ around the equilibrium state up to second order in $x=R-R_{0}$, hence obtaining

$\frac{d^{2} x}{d \tau^{2}}+\omega_{0}^{2} x=0$

Stability implies that

$\left.\omega_{0}^{2} \equiv \frac{1}{2} \frac{d^{2} V}{d R^{2}}\right|_{R_{0}}>0$.

The calculation of $\omega_{0}^{2}$ involves $\frac{d \sigma}{d R}$ (which is given by Eq. $15)$, and $\frac{d^{2} \sigma}{d R^{2}}$, which is obtained by taking the derivative of Eq. (15). For a general EOS of the type $P=P(R, \sigma), \omega_{0}^{2}$ is given by [7]:
The line dividing stability from instability is given by $\omega_{0}^{2}=0$ which, using Eq. (2), leads to the following expression for the critical values of $\Omega_{20}$ :

$\Omega_{20 c}=\frac{A \Omega_{10 c}+B}{D}$

where

$$
\begin{aligned}
A= & \pi R_{0}^{3}\left[8\left(2 R_{0}^{2}+6 m_{0}^{2}-7 R_{0} m_{0}\right) u\right. \\
& \left.+72 R_{0}^{2} m_{0}+32 m_{0}^{3}-96 R_{0} m_{0}^{2}-16 R_{0}^{3}\right] \\
B= & {\left[m_{0}^{2}\left(3 m_{0}-15 R_{0}\right)+R_{0}^{2}\left(14 m_{0}-4 R_{0}\right)\right] u } \\
& \quad+R_{0}\left[m_{0}\left(27 m_{0} R_{0}^{2}-13 m_{0}^{2}-18 R_{0}^{2}\right)+4 R_{0}^{3}\right] \\
D=2 & {\left[R_{0}^{2}\left(4 R_{0}-18 m_{0}\right)+m_{0}^{2}\left(23 R_{0}-6 m_{0}\right)\right] u } \\
& +R_{0}\left[-4 R_{0}^{3}+m_{0}\left(22 R_{0}^{2}-39 m_{0} R_{0}+22 m_{0}^{2}\right)\right]
\end{aligned}
$$

with $u \equiv \sqrt{R_{0}\left(R_{0}-2 m_{0}\right)}$. Equation (22), valid for an arbitrary EOS of the form $P=P(R, \sigma)$, determines regions of stability in a certain space of parameters. In particular, in the barotropic case, $\Omega_{10}=0$, and $\Omega_{20 c}=B / D$ defines the surface $\Omega_{20 c}=\Omega_{20 c}\left(m_{0}, R_{0}\right)$. Any equilibrium configuration with $\left(m_{0}, R_{0}\right)$ such that $\Omega_{20}$ is greater that $\Omega_{20 c}$ will be stable. ${ }^{5}$ In the non-barotropic case, Eq. (22) defines a 3-d surface by $\Omega_{20 c}=\Omega_{20 c}\left(m_{0}, R_{0}, \Omega_{10 c}\right)$.

When a specific EOS is chosen, there are other constraints that must be taken into account. As we shall see in Sect. 4, using the equilibrium equations (4) and (5) for a given EOS, we can obtain the derivatives of the EOS as $\Omega_{10}=\Omega_{10}\left(m_{0}, R_{0} ; \kappa\right)$, and $\Omega_{20}=\Omega_{20}\left(m_{0}, R_{0} ; \kappa\right)$, where $\kappa$ denotes the parameters of the EOS. The EOS and the equilibrium equations also yield $m=m\left(R_{0} ; \kappa\right)$. Combining the latter with the equation (21) for $\Omega_{20}$ we obtain $\Omega_{20}=\Omega_{20}\left(R_{0}^{\prime}\right)$, where $R_{0}^{\prime}$ is the radius of the equilibrium configuration normalized using the dimension-full parameter of the EOS. Using $m=m\left(R_{0} ; \kappa\right)$ and the equation for $\Omega_{10}$ in the Eq. (22) for $\Omega_{20 c}$, we obtain $\Omega_{20 c}=\Omega_{20 c}\left(R_{0}^{\prime}\right)$, The

$$
\begin{aligned}
\omega_{0}^{2}= & \frac{1}{2}\left\{-16 \pi \frac{H_{0} F_{0}}{F_{0}-H_{0}} \Omega_{10}+2 \frac{H_{0}\left(2 F_{0}^{2}-f_{10}^{\prime} R_{0}\right)-F_{0}\left(2 H_{0}^{2}-f_{20}^{\prime} R_{0}\right)}{\left(F_{0}-H_{0}\right) R_{0}^{2}} \Omega_{20}\right. \\
& \left.+\frac{\left[4 F_{0}^{4}-2 R_{0} F_{0}^{2}\left(f_{10}^{\prime}+R_{0} f_{10}^{\prime \prime}\right)+R_{0}^{2} f_{10}^{\prime 2}\right] H_{0}^{3}-\left[4 H_{0}^{2}-2 R_{0} H_{0}^{2}\left(f_{20}^{\prime}+R_{0} f_{20}^{\prime \prime}\right)+R_{0}^{2} f_{20}^{\prime 2}\right] F_{0}^{3}}{2\left(F_{0}-H_{0}\right) F_{0}^{2} H_{0}^{2} R_{0}^{2}}\right\},
\end{aligned}
$$

where $F_{0} \equiv \sqrt{f_{i 0}}, H_{0} \equiv \sqrt{f_{o 0}}$, and

$$
\begin{aligned}
& \left.\Omega_{10} \equiv \frac{d P}{d R}\right|_{R_{0}}, \\
& \left.\Omega_{20} \equiv \frac{d P}{d \sigma}\right|_{R_{0}} .
\end{aligned}
$$

\footnotetext{
5 Equation (22) was used in [7] to analyze the dynamical stability of two systems (a thin shell connecting two spacetimes of cloud of strings, and a thin shell connecting vacuum to Schwarzschild) in the $\left(\Omega_{10 c}, \Omega_{20 c}\right)$ plane without specifying the EOS.
} 
dynamically stable configurations for the given EOS will be those with $\Omega_{20}\left(R_{0}^{\prime}\right)>\Omega_{20 c}\left(R_{0}^{\prime}\right)$. We shall consider in the next section the dynamical stability of the shell presented in Sect. 2 for several relevant examples of EOS.

\section{Dynamical stability for different EOS and the $m_{0}=m_{0}\left(R_{0}\right)$ curve}

Let us apply next the discussion of the previous section to several EOS of interest, to determine the regions of dynamical stability in the $\left(m_{0}, R_{0}\right)$ plane, as well as the actual equilibrium states given by the $m_{0}=m_{0}\left(R_{0}\right)$ curve. It is important to note that the results from the dynamical stability analysis we shall present are in agreement with the criterion of the maximum of the $m_{0}=m_{0}\left(R_{0}\right)$ diagram, a fact that was proved in [11] for the case of a barotropic EOS. Our results suggest that the criterion is also valid for EOS of the form $P=P(R, \sigma)$. All the $m_{0}=m_{0}\left(R_{0}\right)$ curves stop at the point where the DEC $P \leq \sigma$ ceases to be satisfied.

\subsection{Quadratic and barotropic EOS}

We shall start with the example of a barotropic EOS, given by

$$
P=\beta \sigma^{2},
$$

where $[\beta]=L$. Such an equation models the non-relativistic limit of a two-dimensional ideal Fermi gas at $T=0$, discussed in Sect. 4.2. The constant $\beta$ can be used to render dimensionless all the variables in the problem as follows: $\sigma^{*}=\beta \sigma, P^{*}=\beta P, R^{*}=R / \beta$, and $m^{*}=m / \beta$. In these variables, Eq. (23) reads $P^{*}=\sigma^{* 2}$. Substituting $P$ into Eq. (9) we obtain $\sigma_{0}=\sigma_{0}\left(R_{0}\right)$ which, in dimensionless form, is given by

$\sigma_{0}^{*}\left(R_{0}^{*}\right)=\frac{1}{4}\left(\frac{1}{\pi R_{0}^{*}}-1\right)$.

Using $\sigma_{0}^{*}\left(R_{0}^{*}\right)$ in Eq. (11) we obtain the corresponding $m_{0}^{*}\left(R_{0}^{*}\right)$ relation:

$m_{0}^{*}\left(R_{0}^{*}\right)=\frac{1}{2} R_{0}^{*}\left(1-\pi^{2} R_{0}^{* 2}\right)$.

In the domain $R_{0}^{*} \geq 0$, the function $m_{0}^{*}\left(R_{0}^{*}\right)$ has a maximum for $R_{0 \max }^{*}=1 /(\pi \sqrt{3})$, resulting in $m_{0 \max }^{*}=1 /(3 \pi \sqrt{3})$. The $m_{0}^{*}=m_{0}^{*}\left(R_{0}^{*}\right)$ curve for this case is shown in Fig. 1.

In the low energy density limit $\left(\sigma^{*} \rightarrow 0\right)$, we can see that $m^{*} \rightarrow 0$ and $R^{*} \rightarrow 1 / \pi$ (thus indicating that this EOS allows shells with very low mass). In the high energy density limit $\left(\sigma^{*} \rightarrow \infty\right)$, we have $m^{*} \rightarrow 0$ and $R^{*} \rightarrow 0$, however this region of the curve is not depicted because it violates the DEC. Notice also that $m_{0}^{*}\left(R_{0}^{*}\right)$ given by (25) satisfies

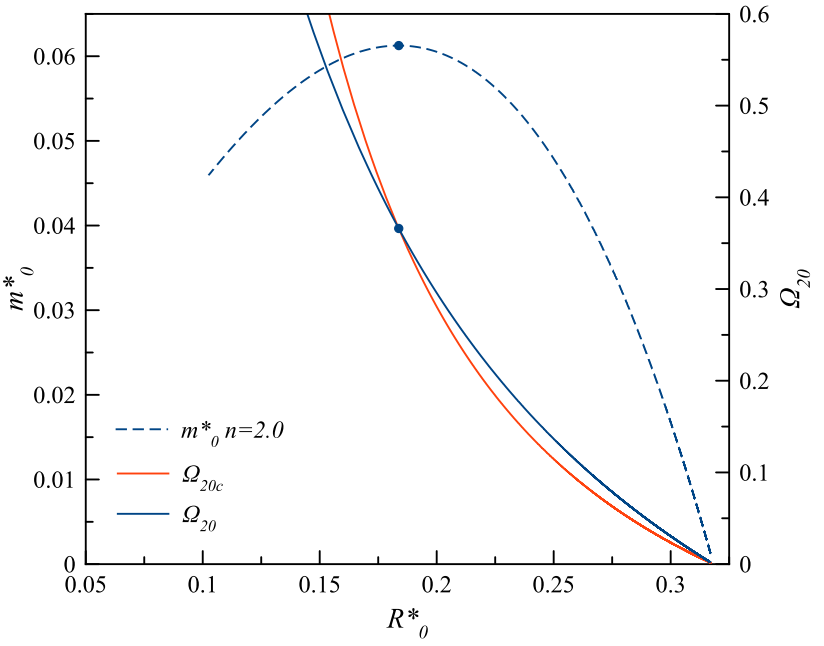

Fig. 1 The figure shows the $m_{0}^{*}\left(R_{0}^{*}\right)$ diagram for the EOS $P=\beta \sigma^{2}$, as well as the critical values of $\Omega_{2}$ that follow from dynamical stability (see Eq. 22), and those that follow from the EOS, in red and blue, respectively

$2 m_{0}^{*}<R_{0}^{*}$, which is a constraint from the theory of thin shells [15].

Next we indicate how to obtain the curves for $\Omega_{20 c}$ and $\Omega_{20}$ of Sect. 3. Using Eqs. (25) in (22) with $\Omega_{10 c}=0$, the curve $\Omega_{20 c}=\Omega_{20 c}\left(R_{0}^{*}\right)$ follows, plotted in red in Fig. 1. For $\Omega_{20}$ we use that $\left.\Omega_{20} \equiv \frac{d P}{d \sigma}\right|_{0}=2 \sigma_{0}^{*}$, where $\sigma_{0}^{*}$ is given by Eq. (24). This yields $\Omega_{20}=\Omega_{20}\left(R_{0}^{*}\right)$, plotted in blue in Fig. 1 . The curve $\Omega_{20}\left(R_{0}^{*}\right)$ intersects $\Omega_{20 c}\left(R_{0}^{*}\right)$ exactly at the value of $R_{0}^{*}$ corresponding to the maximum of the $m_{0}^{*}\left(R_{0}^{*}\right)$ curve, and all the configurations on the curve to the right of this point $\left(\Omega_{20}>\Omega_{20 c}\right)$ are stable.

\subsection{A relativistic EOS (EOS I)}

As shown in the Appendix, the EOS for a system of noninteracting relativistic fermions in $2 \mathrm{~d}$ at $T=0$ is given by

$\sigma_{0}=\frac{\alpha}{3 \pi}\left[\left(x^{2}+1\right)^{3 / 2}-1\right]$,

$P_{0}=\frac{\alpha}{3 \pi}\left[\frac{1}{2}\left(x^{2}-2\right) \sqrt{x^{2}+1}+1\right]$,

with $x=p_{F} / m c, \alpha=m c^{2} / \lambda^{2}$ and $\lambda=\hbar / m c$. Following the same steps as in Sect. 4.1, where the dimensionless variables are now $\sigma^{*}=\sigma / \alpha, P^{*}=P / \alpha, R^{*}=\alpha R$, and $m^{*}=\alpha m$, we obtain the plots shown in Fig. 2 .

The values to the right of the maximum at $\left(R_{0 \max }^{*}, m_{0 \max }^{*}\right)$ $=(0.26545,0.060666)$ are stable. In the low energy density limit $\left(\sigma^{*} \rightarrow 0\right)$, the plots show that $m^{*} \rightarrow 0$ and $R^{*} \rightarrow 0.5$ (indicating that this EOS also allows shells with very low mass), while in the high energy density limit $\left(\sigma^{*} \rightarrow \infty\right)$, 


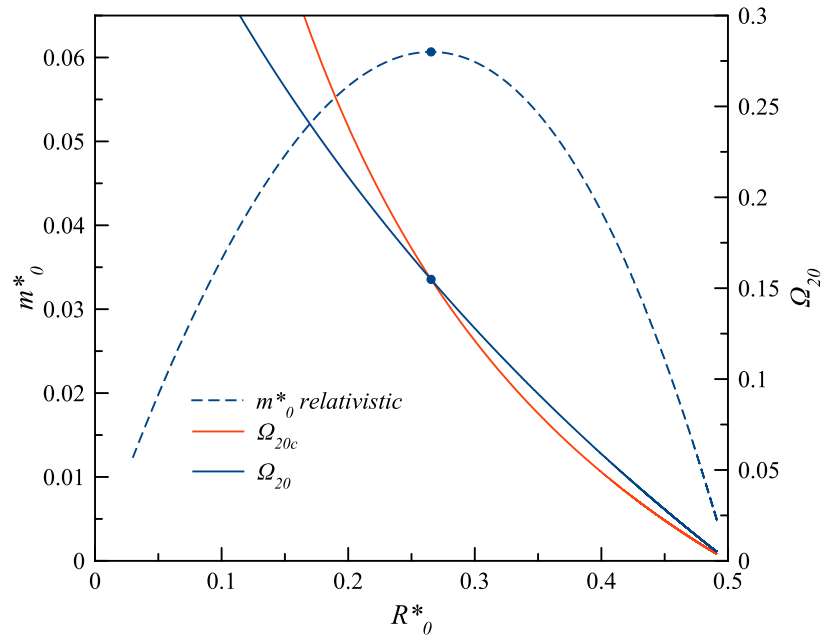

Fig. 2 The figure shows the $m_{0}^{*}=m_{0}^{*}\left(R_{0}^{*}\right)$ diagram for the relativistic EOS defined by Eqs. (26) and (27), as well as the critical values of $\Omega_{2}$ that follow from dynamical stability and those that follow from the EOS, in red and blue, respectively

$m^{*} \rightarrow 0$ and $R^{*} \rightarrow 0$. The DEC is satisfied along the whole curve as expected, together with the constraint $2 m_{0}^{*}<R_{0}^{*}$.

\subsection{A more general barotropic EOS (EOS II)}

Let us to study now the EOS II given by

$P=\beta \sigma^{n}$,

where $[\beta]=L^{n-1}$. The case $n=2$ corresponds to the case studied in Sect. 4.1. Equations (9) and (11) now read

$$
\begin{aligned}
R_{0}\left(\sigma_{0}\right) & =\frac{\beta \sigma_{0}^{n-2}}{\pi\left(1+4 \beta \sigma_{0}^{n-1}\right)}, \\
m_{0}\left(\sigma_{0}\right) & =4 \pi \sigma R_{0}^{2}\left(1-2 \pi \sigma_{0} R_{0}\right) .
\end{aligned}
$$

Defining the dimensionless quantities $\sigma^{*}, P^{*}, R^{*}$ and $m^{*}$ by

$$
\begin{aligned}
& \sigma^{*}=\beta^{\frac{1}{n-1}} \sigma, \\
& P^{*}=\beta^{\frac{1}{n-1}} P, \\
& R^{*}=\beta^{\frac{1}{1-n}} R, \\
& m^{*}=\beta^{\frac{1}{1-n}} m,
\end{aligned}
$$

equations (28), (29) and (30) read

$$
\begin{aligned}
& P_{0}^{*}=\sigma_{0}^{* n}, \\
& R_{0}^{*}=\frac{\sigma_{0}^{* n} n-2}{\pi\left(1+4 \sigma_{0}^{*(n-1)}\right)}, \\
& m_{0}^{*}=4 \pi \sigma_{0}^{*} R_{0}^{* 2}\left(1-2 \pi \sigma_{0}^{*} R_{0}^{*}\right) .
\end{aligned}
$$

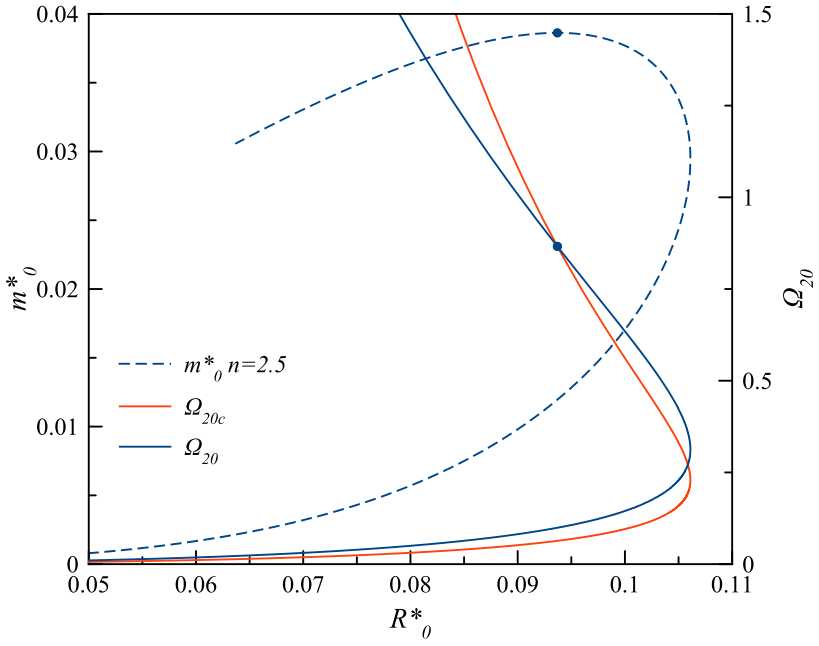

Fig. 3 The figure shows the $m_{0}^{*}=m_{0}^{*}\left(R_{0}^{*}\right)$ diagram for the EOS $P=$ $\beta \sigma^{2.5}$, as well as the curves for $\Omega_{20 c}$ and $\Omega_{20}$

Equations (36) and (37) can be solved numerically to build the $m_{0}^{*}=m_{0}^{*}\left(R_{0}^{*}\right)$ curves, an example of which is shown in Fig. 3 (for $n=2.5$ ). The curves corresponding to $\Omega_{20} c$ and $\Omega_{20}$ are also shown. The stability interval goes from the maximum of the $m_{0}^{*}=m_{0}^{*}\left(R_{0}^{*}\right)$ curve (which again coincides with the crossing of the $\Omega_{20}$ curves), all the way down to small values of $m_{0}^{*}$ and $R_{0}^{*}$.

The upper panel of Fig. 4 shows the $m_{0}^{*}=m_{0}^{*}\left(R_{0}^{*}\right)$ curves for $n=3.0,3.5,4.0$ (dashed line), the associated $k=k\left(R_{0}\right)$ curves (Eq. 12) for each case (full line), and the smallest value of $k\left(k_{\min }=1 / 5\right)$ which satisfies the DEC (these curves will be useful below). The $\Omega_{20}$ and $\Omega_{20}$ curves are displayed in the lower panel. In all cases, the value of $R_{0}^{*}$ corresponding to the maximum of the $m_{0}^{*}=m_{0}^{*}\left(R_{0}^{*}\right)$ curve coincides with that of the crossing of the $\Omega_{2}$ curves. Also, all cases approach $m^{*}=R^{*}=0$ for $\sigma^{*} \rightarrow \infty$ (high-energy limit, not shown due to the DEC violation) and $\sigma^{*} \rightarrow 0$ (low-energy limit), and verify the condition $2 m_{0}^{*}<R_{0}^{*}$. Notice also that in the dimensionless quantities we are using, all the $m_{0}^{*}=m_{0}^{*}\left(R_{0}^{*}\right)$ curves end at the same point, where the DEC is marginally satisfied (i.e. $P_{0}^{*}=\sigma_{0}^{*}$ ).

\section{A non-barotropic EOS (EOS III)}

In this section we shall explore the dynamically stable equilibrium configurations that follow from the non-barotropic EOS given by [23]

$P(R, \sigma)=\frac{A}{R^{n}} \sigma$

where $[A]=L^{n}$. Defining for convenience the dimensionless energy density, pressure, radius and mass as 

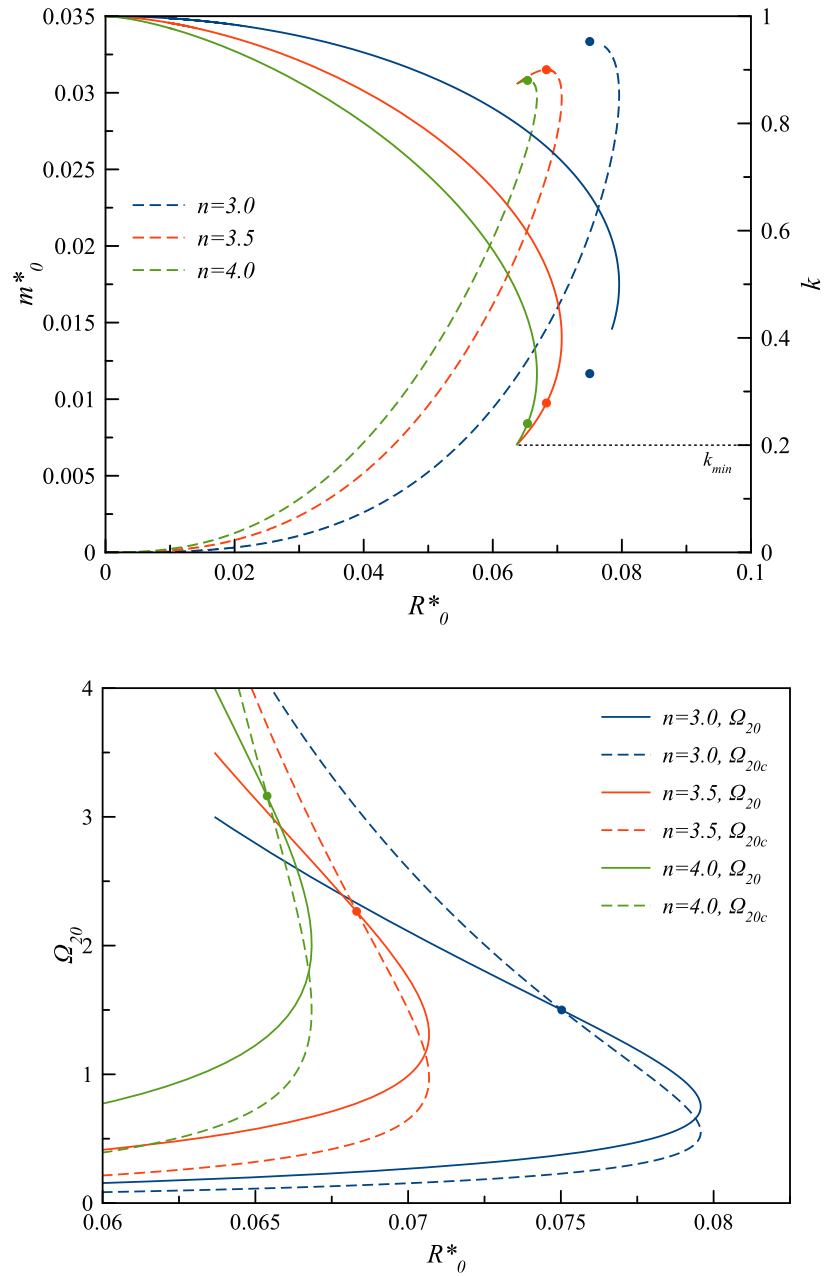

Fig. 4 Upper panel: $m_{0}^{*}=m_{0}^{*}\left(R_{0}^{*}\right)$ (dashed line) and $k=k\left(R_{0}^{*}\right)$ curves (full line) for EOS II and several values of $n . k_{\min }=1 / 5$ is the smallest value of $k$ which satisfies the DEC. Lower panel: plots of $\Omega_{20}$ and $\Omega_{20 c}$ in terms of $R_{0}^{*}$ for different values of $n$ in EOS II. Regarding $m_{0}^{*}$ and $\Omega_{20}$, note that the value of the $R_{0}^{*}$ coordinate of each of the coloured dots in the upper and lower panels is the same

$\sigma^{*}=\sigma A^{1 / n}$,

$P_{0}^{*}=P A^{1 / n}$,

$R^{*}=\frac{R}{A^{1 / n}}$

$m^{*}=\frac{m}{A^{1 / n}}$,

and using the EOS in the equations for the mechanical equilibrium of the shell, given in Eqs. (9) and (11), the following $m_{0}^{*}\left(R_{0}^{*}\right)$ relation is obtained:

$m_{0}^{*}\left(R_{0}^{*}\right)=\frac{4 R_{0}^{*}\left(R_{0}^{* n}+2\right)}{\left(R_{0}^{* n}+4\right)^{2}}$.

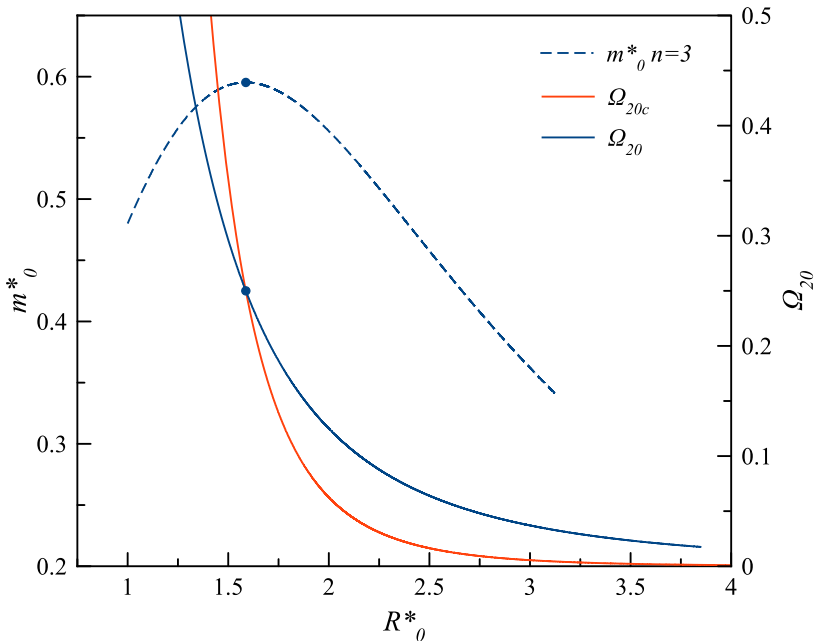

Fig. $5 m_{0}^{*}=m_{0}^{*}\left(R_{0}^{*}\right)$ diagram, as well as $\Omega_{20}$ and $\Omega_{20 c}$ curves for EOS III with $n=3$

The extremum is given at

$R_{0 m}^{*}=\left(\frac{3+\sqrt{8 n+1}}{n-1}\right)^{1 / n} \quad$, for $n>1$

The maximum in the $m_{0}^{*}=m_{0}^{*}\left(R_{0}^{*}\right)$ diagram, is given by

$$
\begin{aligned}
m_{0 \max }^{*}=m_{0}^{*}\left(R_{0 m}^{*}\right)= & 4(n-1)\left(\frac{3+\sqrt{8 n+1}}{n-1}\right)^{1 / n} \\
& \left(\frac{\sqrt{8 n+1}+2 n+1}{\sqrt{8 n+1}+4 n-1}\right), \quad n>1 .
\end{aligned}
$$

The $m_{0}^{*}=m_{0}^{*}\left(R_{0}^{*}\right)$ curve for $n=3$ is shown in Fig. 5 .

The stable equilibrium configurations are those located to the right of the maximum, in correlation with the crossing of the $\Omega_{2}$ curves. Notice that there are configurations with very small $m_{0}^{*}$ and very large $R_{0}^{*}$. The $m_{0}^{*}=m_{0}^{*}\left(R_{0}^{*}\right)$ curves for several values of $n$ are shown in the upper panel of Fig. 6 (dashed line) together with the corresponding $k\left(R_{0}^{*}\right)$ curves (full line), while the associated $\Omega_{2}$ curves are displayed in the lower panel. In all cases, the value of $R_{0}^{*}$ at the maximum of $m_{0}^{*}=m_{0}^{*}\left(R_{0}^{*}\right)$ coincides with that of the crossing of the $\Omega_{2}$ curves.

\section{Thermodynamical stability}

We shall compare next the results presented in the previous sections (via dynamical stability) with those originating from thermodynamical stability. This type of stability was studied for the neutral shell in [20]. Two equations of state were used there, a phenomenological one for the temperature, and the 

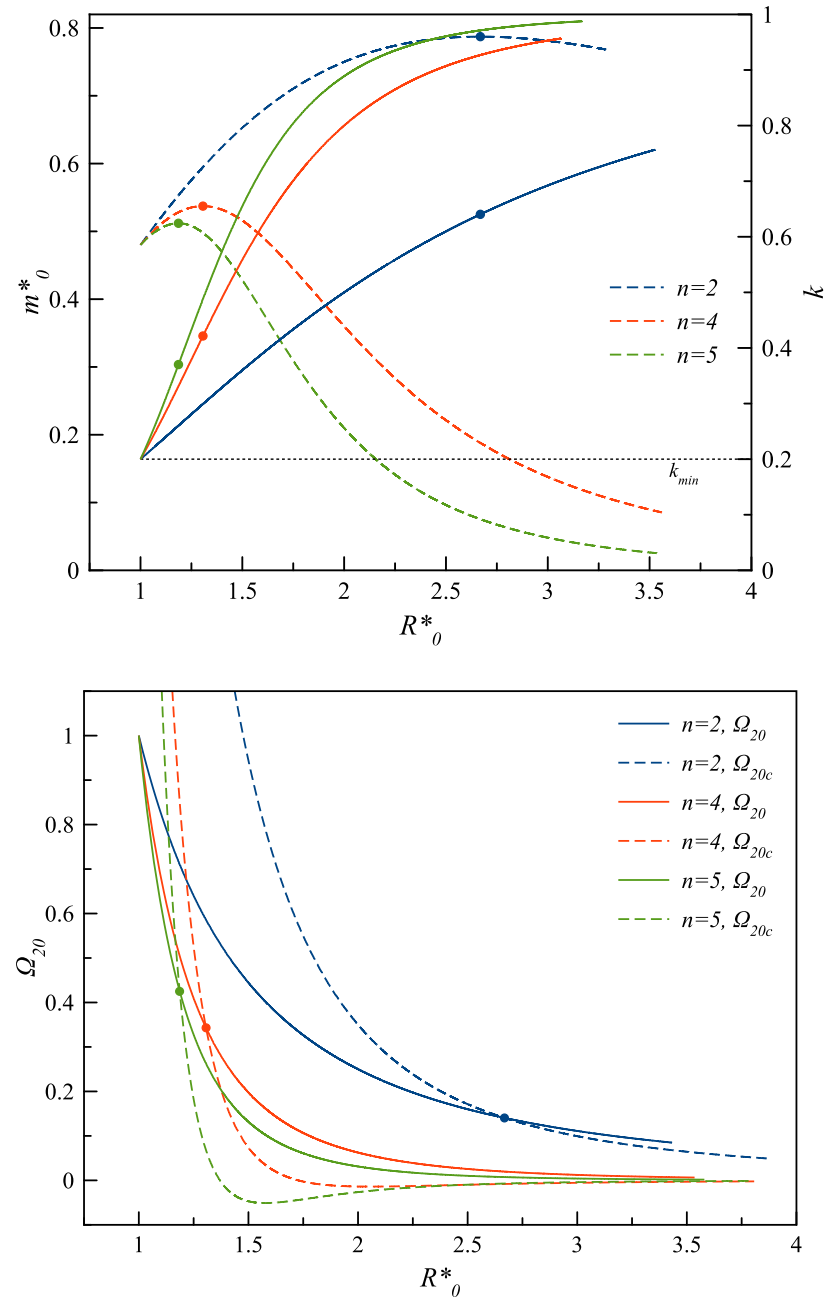

Fig. 6 Upper panel: The figure shows the $m_{0}^{*}=m_{0}^{*}\left(R_{0}^{*}\right)$ (dashed line) and $k=k\left(R_{0}^{*}\right)$ curves (full line) for EOS III and several values of $n$. $k_{\min }=1 / 5$ is the smallest value of $k$ which satisfies the DEC. Lower panel: $\Omega_{20}$ and $\Omega_{20 c}$ curves for different values of $n$ for the non-barotropic EOS III. As in Fig. 4 regarding $m_{0}^{*}$ and $\Omega_{20}$, the value of the $R_{0}^{*}$ coordinate of each of the coloured dots in the upper and lower panels is the same

one that follows from the junction conditions (Eq. (7)), to obtain the entropy of a thin shell with constant number of particles. Starting from the first law:

$d S=\beta d M+p d A$

where $\beta \equiv 1 / T$ and $A \equiv 4 \pi R^{2}$, Martinez showed that, as a consequence of Eq. (7) and the integrability condition for $S$, the function $\beta$ must have the form

$\beta(M, R)=b\left(r_{+}\right) k$,

where $b$ is an arbitrary function of $r_{+}\left(M, R_{0}\right)=2 m\left(M, R_{0}\right)$, and $k$ is given in Eq. (12). The explicit form of the function $b$ should be obtained from an explicit model of the matter that composes the shell. The following phenomenological form was chosen in [20]:

$b\left(r_{+} ; \eta, a\right)=\frac{2 \eta}{\ell_{P} 1+a} r_{+}^{a}$,

where $\eta$ and $a$ are dimensionless coefficients. Such a choice leads to

$S\left(M, R_{0} ; \eta, a\right)=\frac{\eta}{1+a}\left(\frac{r_{+}}{\ell_{P}}\right)^{1+a}+S_{0}$,

for $a \neq-1$. This expression reduces to the BekensteinHawking entropy for $a=1$ and $\eta=2 \pi$. By demanding that a zero mass shell possess null entropy, it follows that $S_{0}=0$ and $a>-1$.

The regions of thermodynamical stability in the $(M, R)$ plane are determined by the conditions

$$
\begin{aligned}
\left(\frac{\partial^{2} S}{\partial M^{2}}\right)_{A} \leq 0, \\
\left(\frac{\partial^{2} S}{\partial A^{2}}\right)_{M} \leq 0, \\
\left(\frac{\partial^{2} S}{\partial M^{2}}\right)_{A}\left(\frac{\partial^{2} S}{\partial A^{2}}\right)_{M}-\left(\frac{\partial^{2} S}{\partial M \partial A}\right)^{2} \geq 0 .
\end{aligned}
$$

As shown in [20], these conditions are more concisely expressed in terms of $k$. Together with the normalization of the entropy and the DEC, they imply that

$-1<a \leq \frac{12}{19}$

for a stable shell. For $-1<a \leq 0$ the stability conditions do not constrain the value of $k$, hence the sole constraint for stability is that coming from the dominant energy condition, namely $R \geq \frac{25}{24} r_{+}$, or $k \geq 1 / 5$. The stability condition with the crossed derivatives leads to

$(3 a+1) k^{2}+2 a k+(a-1) \leq 0$,

which is automatically satisfied for $a \leq 0$, but restricts the values of $k$ for $0<a \leq 12 / 19$.

\section{Dynamical and thermodynamical stability}

Before presenting the results for all the EOS considered here with the Ansatz in Eq. (48), let us give an example. Figure 7 shows the $m_{0}^{*}=m_{0}^{*}\left(R_{0}^{*}\right)$ diagram for the EOS II with $n=2$, and the values of $k$ obtained from its definition, using the equilibrium curve $m_{0}^{*}=m_{0}^{*}\left(R_{0}^{*}\right)$.

For the case at hand, such a curve is given by Eq. (25), which results in $k\left(R_{0}^{*}\right)=\pi R_{0}^{*}$. The points that are dynamically stable are those on the curve to the right of $A_{m^{*}}$, 


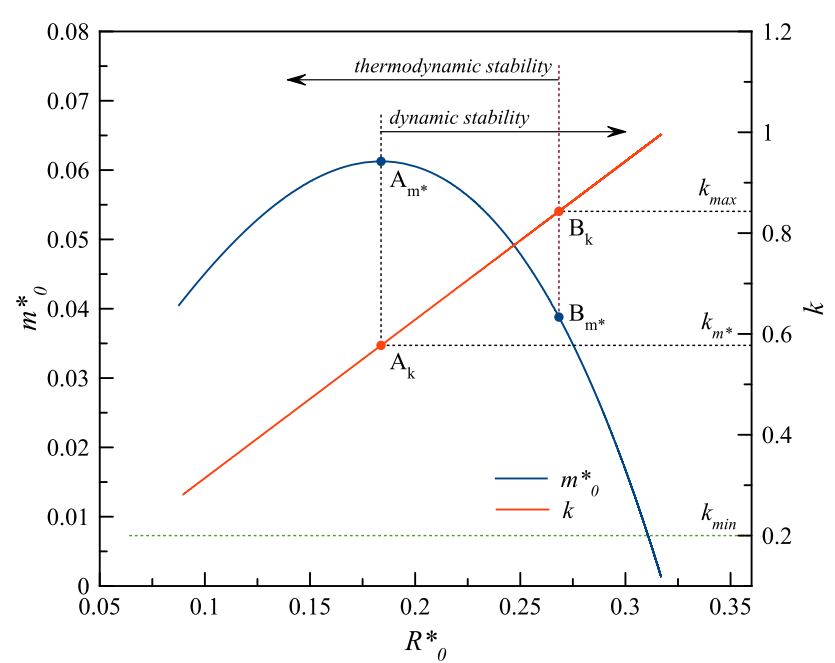

Fig. 7 Dynamic and thermodynamic stability regions for EOS II with $n=2$ and $a=0.06$. The states with $k<k_{\min }=1 / 5$ (which corresponds to $a>a_{\max }=12 / 19$ ) violate the DEC. The allowed values of $k$ for thermodynamic stability satisfy $k_{\min }<k<k_{\max }$, where $k_{\max }=0.843130$ follows from Eq. (53). The allowed values of $k$ for dynamical stability satisfy $k_{m^{*}}<k$, where $k_{m^{*}}=1 / \sqrt{3}$ corresponds to the maximum of the curve $m_{0}^{*}=m_{0}^{*}\left(R_{0}^{*}\right)$

which correspond to the interval $k>k_{m^{*}}=1 / \sqrt{3}$. The thermodynamical stability region is $k_{\min }<k<k_{\max }$, where $k_{\max }=0.843130$ is determined by Eq. (53) with $a=0.06$. Hence, the states that are both thermodynamically and dynamically stable are those between the points $A_{m^{*}}$ and $B_{m^{*}}$ in the $m_{0}^{*}=m_{0}^{*}\left(R_{0}^{*}\right)$ curve. Figure 8 presents the region in the $(a, k)$ plane which correspond to configurations that are thermodynamically stable (which are those between the horizontal line $k_{\min }$ and the curve $k_{\max }=k_{\max }(a)$ ), as well as that of dynamically stable configurations (which are those above the horizontal line $k_{m^{*}}$ ). The intersection of these regions yields the set of points $(a, k)$ associated to configurations that are both thermodynamically and dynamically stable.

Figure 9 presents the results in the $(a, k)$ plane for all the EOS we have examined.

For a given value of $a$, the thermodynamically stable configurations are those with $k_{\min }<k<k_{\max }$. Also shown in the plot are the points at which the horizontal line associated to the value of $k$ corresponding to the maximum of the $m_{0}^{*}=m_{0}^{*}\left(R_{0}^{*}\right)$ curve $\left(k_{m^{*}}\right)$ intersects the $k_{\max }=k_{\max }(a)$ curve, obtained from Eq. (53). For a given EOS, the configurations that are both thermodynamically and dynamically stable are those above the horizontal line that crosses the corresponding point and below the $k_{\max }=k_{\max }(a)$ curve. The plot clearly shows that the requirement of thermodynamical stability greatly restricts the region of the parameter space allowed by the dynamical stability. It also follows from our results that the region associated to dynamical stability is larger for larger values of $n$, for both types of EOS consid-

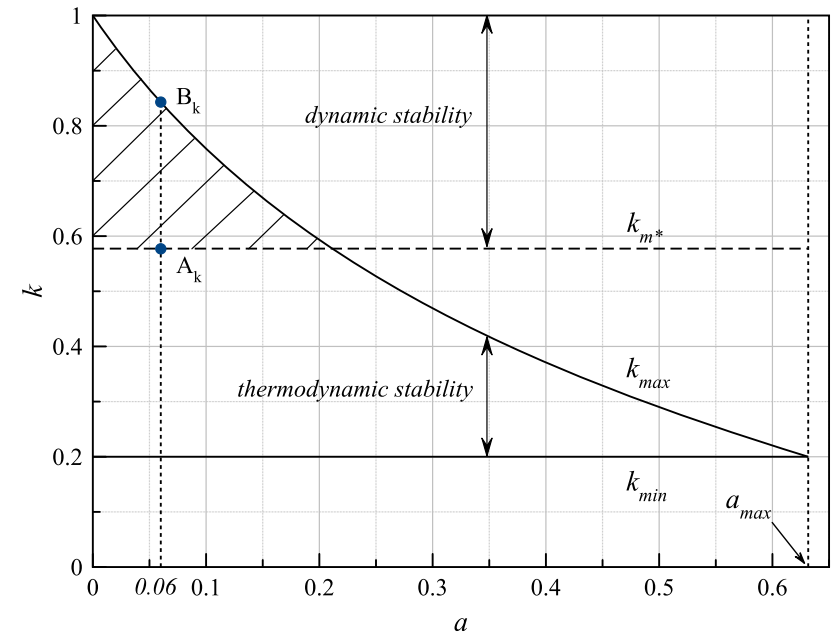

Fig. 8 Dynamic and thermodynamic stability for EOS II with $n=2$ in the $(a, k)$ plane. The curve $k_{\max }(a)$ follow from Eq. (53). The points $A_{k}$ and $B_{k}$ are those in Fig. 7

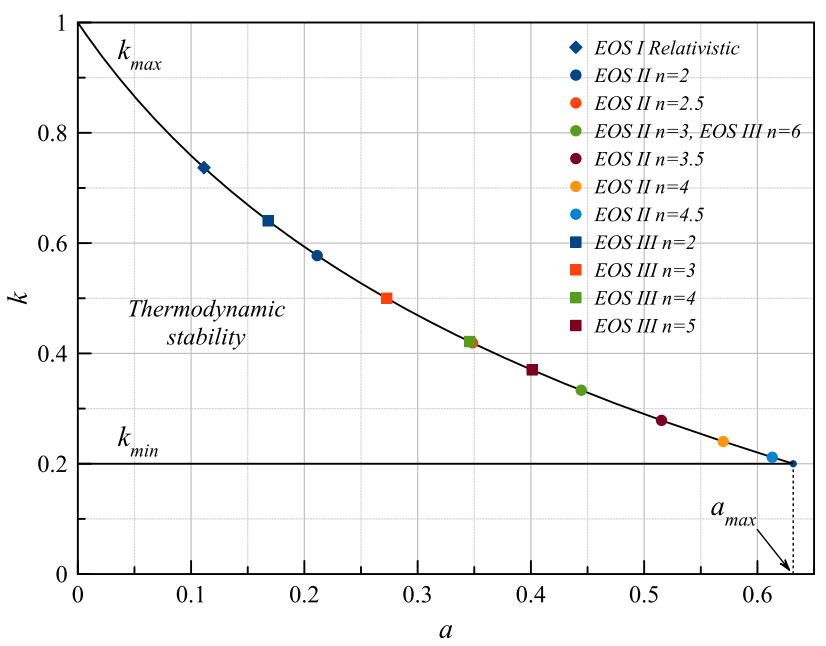

Fig. 9 Dynamic and thermodynamic stability for all the EOS examined here. The states with $k<k_{\text {min }}=1 / 5$ (which corresponds to $\left.a>a_{\max }=12 / 19\right)$ violate the DEC. The allowed values of $k$ for thermodynamic stability are those below the $k_{\max }(a)$ curve, determined by Eq. 53, and above $k_{\min }$. The allowed values of $k$ for dynamical stability are those above the value of $k$ on the curve for each EOS, which corresponds to the value of $k_{m^{*}}$ of the maximum of the curve $m_{0}^{*}=m_{0}^{*}\left(R_{0}^{*}\right)$ for each EOS

ered here. This is to be expected since a larger $n$ is associated with stiffer matter.

\section{Closing remarks}

We have examined the dynamical and thermodynamical stability of neutral mass shells for different equations of state, described by $P=P(R, \sigma)$. To attain this goal, the condition for dynamical stability valid for an arbitrary EOS (obtained in previous works) given in terms of the derivatives $\Omega_{1}=\frac{d P}{d R}$ 
and $\Omega_{2}=\frac{d P}{d \sigma}$ was adapted to the case of a given EOS, yielding the curve $\Omega_{20 c}=\Omega_{20 c}\left(R_{0}^{*}\right)$. The result was compared to the $\Omega_{2}=\Omega_{2}\left(R_{0}^{*}\right)$ curve that follows from the given EOS and the equations for a shell in equilibrium, to determine the dynamically stable configurations. Using the criteria for thermodynamical stability and a specific form for the entropy of the shell, the set of thermodynamically stable configurations was also determined. The main result is that thermodynamical stability greatly constraints set of stable equilibrium configurations determined by dynamical stability, due to the upper constraint defined by Eq. (53). Our results also confirm that stable states are those to the right of the maximum of the $m_{0}^{*}=m_{0}^{*}\left(R_{0}^{*}\right)$ curve. Such a link between the maximum mass along a sequence of equilibrium configurations of the shell and the onset of stability was obtained in [11] for the case of a barotropic EOS. Our findings suggest that it can be extended to EOS of the type $P=P(R, \sigma)$.

The generalization and consequences of our results to the interesting cases of self-gravitating shells in $d$ dimensions [1], and charged [15] and rotating [13] shells are left for future work.

Acknowledgements This work was supported by PROSNI 2018-2019, Conacyt México and Universidad de Guadalajara.

Data Availability Statement This manuscript has no associated data or the data will not be deposited. [Authors' comment: This manuscript is a theoretical study and no experimental data is associated to it.]

Open Access This article is licensed under a Creative Commons Attribution 4.0 International License, which permits use, sharing, adaptation, distribution and reproduction in any medium or format, as long as you give appropriate credit to the original author(s) and the source, provide a link to the Creative Commons licence, and indicate if changes were made. The images or other third party material in this article are included in the article's Creative Commons licence, unless indicated otherwise in a credit line to the material. If material is not included in the article's Creative Commons licence and your intended use is not permitted by statutory regulation or exceeds the permitted use, you will need to obtain permission directly from the copyright holder. To view a copy of this licence, visit http://creativecomm ons.org/licenses/by/4.0/.

Funded by SCOAP ${ }^{3}$.

\section{Appendix: 2d fermion gas}

We obtain here the EOS of a two-dimensional relativistic ideal Fermi gas of $N$ particles of mass $m$ at $T=0$ in a square of side $L$. In the thermodynamical limit we have $L \rightarrow$ $\infty, N \rightarrow \infty$ and the particle number density $n=N / L^{2}$ constant.

For the summation over states $q$, we have in the continuous case

$\sum_{q} \longrightarrow \frac{2 L^{2}}{h^{2}} \int d^{2} p$ where $p$ is the two-dimensional linear momentum. Then, for the particle number density $n=N / L^{2}$ we have

$$
\begin{aligned}
N & =\frac{2 L^{2}}{h^{2}} \int_{0}^{p_{F}} d^{2} p=\frac{L^{2}}{2 \pi \hbar^{2}} p_{F}^{2}, \\
n & =\frac{p_{F}^{2}}{2 \pi \hbar^{2}}=\frac{x^{2}}{2 \pi \lambda^{2}},
\end{aligned}
$$

where $p_{F}$ is the Fermi momentum, $x=p_{F} / m c$, and $\lambda=$ $\hbar / m c$ is the Compton wavelength.

Now we obtain the internal energy density $\sigma$. For the internal energy $U$ of the gas we have

$$
\begin{aligned}
& U=\frac{2 L^{2} 2 \pi}{h^{2}} \int_{0}^{p_{F}} \sqrt{p^{2} c^{2}+m^{2} c^{4}} p d p \\
& \sigma=U / L^{2}=\frac{1}{\pi \hbar^{2}} \int_{0}^{p_{F}} \sqrt{p^{2} c^{2}+m^{2} c^{4}} p d p \\
& \sigma=\frac{m c^{2}}{3 \pi \lambda^{2}}\left[\left(x^{2}+1\right)^{3 / 2}-1\right] .
\end{aligned}
$$

For the pressure we have

$$
\begin{aligned}
& P=\frac{1}{2} \frac{1}{\pi \hbar^{2}} \int_{0}^{p_{F}} \frac{p^{2} c^{2}}{\sqrt{p^{2} c^{2}+m^{2} c^{4}}} p d p, \\
& P=\frac{m c^{2}}{3 \pi \lambda^{2}}\left[\frac{1}{2}\left(x^{2}-2\right) \sqrt{x^{2}+1}+1\right] .
\end{aligned}
$$

Finally, the EOS for a two-dimensional ideal relativistic Fermi gas at $T=0$ follows from Eqs. (54), (55), and (56).

\section{References}

1. R. Andréé, J.P. Lemos, G.M. Quinta, Thermodynamics and entropy of self-gravitating matter shells and black holes in $d$ dimensions. Phys. Rev. D 99(12), 125,013 (2019). https://doi.org/10.1103/ PhysRevD.99.125013

2. P.R. Brady, J. Louko, E. Poisson, Stability of a shell around a black hole. Phys. Rev. D 44, 1891-1894 (1991). https://doi.org/10.1103/ PhysRevD.44.1891

3. J. Crisóstomo, R. Olea, Hamiltonian treatment of the gravitational collapse of thin shells. Phys. Rev. D 69(10), (2004). https://doi.org/ 10.1103/physrevd.69.104023

4. U. Debnath, Variable modified Chaplygin gas and accelerating universe. Astrophys. Space Sci. 312, 295-299 (2007). https://doi.org/ 10.1007/s10509-007-9690-6

5. N.M. Garcia, F.S.N. Lobo, M. Visser, Generic spherically symmetric dynamic thin-shell traversable wormholes in standard general relativity. Phys. Rev. D 86, 044,026 (2012). https://doi.org/10. 1103/PhysRevD.86.04402610.1103/PhysRevD.86.044026

6. Z.K. Guo, Y.Z. Zhang, Cosmology with a variable Chaplygin gas. Phys. Lett. B 645, 326-329 (2007). https://doi.org/10.1016/j. physletb.2006.12.063

7. S. Habib Mazharimousavi, M. Halilsoy, S.N. Hamad Amen, Stability of spherically symmetric timelike thin-shells in general relativity with a variable equation of state. Int. J. Mod. Phys. D 26(14), 175,0158 (2017). https://doi.org/10.1142/S0218271817501589 
8. W. Israel, Singular hypersurfaces and thin shells in general relativity. Il Nuovo Cimento B Ser. 10 44(1), 1-14 (1966). https://doi. org/10.1007/BF02710419

9. J. Kijowski, G. Magli, D. Malafarina, Relativistic dynamics of spherical timelike shells. Gen. Relativ. Gravit. 38(11), 1697-1713 (2006). https://doi.org/10.1007/s10714-006-0323-0

10. H.C. Kim, Physics at the surface of a star in Eddington-inspired Born-Infeld gravity. Phys. Rev. D 89, 064001 (2014). https://doi. org/10.1103/PhysRevD.89.064001

11. P. LeMaitre, E. Poisson, Equilibrium and stability of thin spherical shells in Newtonian and relativistic gravity. Am. J. Phys. 87(12), 961 (2019). https://doi.org/10.1119/10.0000026

12. J.P. Lemos, O.B. Zaslavskii, Entropy of extremal black holes from entropy of quasiblack holes. Phys. Lett. B 695(1-4), 37-40 (2011). https://doi.org/10.1016/j.physletb.2010.11.033

13. J.P.S. Lemos, F.J. Lopes, M. Minamitsuji, J.V. Rocha, Thermodynamics of rotating thin shells in the BTZ spacetime. Phys. Rev. D 92(6), 064,012 (2015). https://doi.org/10.1103/PhysRevD.92. 064012

14. J.P.S. Lemos, M. Minamitsuji, O.B. Zaslavskii, Unified approach to the entropy of an extremal rotating BTZ black hole: thin shells and horizon limits. Phys. Rev. D 96(8), 084,068 (2017). https://doi. org/10.1103/PhysRevD.96.08406810.1103/PhysRevD.96.084068

15. J.P.S. Lemos, G.M. Quinta, O.B. Zaslavski, Entropy of a selfgravitating electrically charged thin shell and the black hole limit. Phys. Rev. D 91(10), 104,027 (2015). https://doi.org/10.1103/ PhysRevD.91.104027

16. J.P.S. Lemos, G.M. Quinta, O.B. Zaslavskii, Entropy of an extremal electrically charged thin shell and the extremal black hole. Phys. Lett. B 750, 306-311 (2015). https://doi.org/10.1016/j.physletb. 2015.08.065
17. J.P.S. Lemos, G.M. Quinta, O.B. Zaslavskii, Entropy of extremal black holes: horizon limits through charged thin shells in a unified approach. Phys. Rev. D 93(8), 084,008 (2016). https://doi.org/10. 1103/PhysRevD.93.084008

18. J.P.S. Lemos, O.B. Zaslavskii, Quasi black holes: definition and general properties. Phys. Rev. D 76, 084,030 (2007). https://doi. org/10.1103/PhysRevD.76.084030

19. P. Martin Moruno, N. Montelongo Garcia, F.S. Lobo, M. Visser, Generic thin-shell gravastars. JCAP 03, 034 (2012). https://doi.org/ 10.1088/1475-7516/2012/03/034

20. E.A. Martinez, Fundamental thermodynamical equation of a selfgravitating system. Phys. Rev. D 53, 7062-7072 (1996). https:// doi.org/10.1103/PhysRevD.53.7062

21. F. Rahaman, M. Kalam, S. Chakraborty, Wormholes with varying equation of state parameter. Acta Phys. Polon. B 40, 25-40 (2009)

22. Roupas, Z.: Thermodynamical instabilities of perfect fluid spheres in general relativity. Class. Quant. Grav. 30(11), 115,018 (2013). https://doi.org/10.1088/0264-9381/32/11/119501.[Erratum: Class.Quant.Grav. 32, 119501 (2015)]

23. V. Varela, Note on linearized stability of Schwarzschild thin-shell wormholes with variable equations of state. Phys. Rev. D 92, 044,002 (2015). https://doi.org/10.1103/PhysRevD.92.044002

24. J.W. York Jr., Black hole thermodynamics and the Euclidean Einstein action. Phys. Rev. D 33, 2092-2099 (1986). https://doi.org/ 10.1103/PhysRevD.33.2092 